\title{
GABAergic mechanisms of anterior and ventromedial hypothalamic nuclei in the expression of freezing in response to a light-conditioned stimulus
}

\author{
Julia Maria dos Santos ${ }^{1}$ and Marcus Lira Brandão ${ }^{2}$ \\ 1 - Instituto de Neurociências \& Comportamento - INeC, Ribeirão Preto, SP, Brasil \\ 2 - Universidade de São Paulo, Ribeirão Preto, SP, Brasil
}

\begin{abstract}
The amygdala, dorsal periaqueductal gray (dPAG), and medial hypothalamus have long been recognized to comprise a neural system responsible for the generation and elaboration of unconditioned fear in the brain. This neural substrate is well known to be under tonic inhibitory control exerted by $\gamma$-aminobutyric acid (GABA) mechanisms. Some evidence also suggests that these structures integrate conditioned fear. A recent study using the fear-potentiated startle paradigm showed that GABAergic mechanisms in the anterior hypothalamic nucleus (AHN) and dorsomedial part of the ventromedial hypothalamic nucleus (VMHDM) regulate conditioned fear. The present study examined the extent to which GABAergic mechanisms in these brain regions are involved in

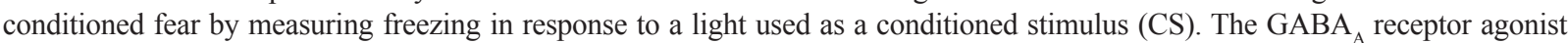
muscimol and the GABA-synthesizing enzyme glutamic acid decarboxylase inhibitor semicarbazide were used as an enhancer and inhibitor of GABA mechanisms, respectively. Muscimol and semicarbazide were injected into the AHN or VMHDM of rats before fear conditioning. Muscimol injections into the AHN and VMHDM significantly reduced conditioned freezing, whereas inhibition of GABA transmission increased this conditioned response in the AHN. The present study further supports the hypothesis that GABAergic mechanisms in the AHN and VMHDM exert inhibitory control on the neural substrates of conditioned fear in the hypothalamus. Keywords: ventromedial hypothalamus, anterior hypothalamus, freezing, GABA.
\end{abstract}

Received 27 August 2011; received in revised form 17 September 2011; accepted 17 September 2011. Available on line 10 October 2011

\section{Introduction}

The medial hypothalamus, amygdala, and dorsal periaqueductal gray ( $\mathrm{dPAG})$ have long been recognized to comprise a neural system responsible for the generation and elaboration of aversive states in the brain (Brandão, Anseloni, Pandóssio, De Araújo, \& Castilho, 1999; Graeff, 2004). Anatomical studies have revealed that these structures are heavily interconnected, suggesting a hierarchically organized brain defense system (Canteras, 2002; Canteras, Chiavegatto, Ribeiro do Valle, \& Swanson, 1997; Graeff, 2004). Particularly interesting for the present study is that the medial zone of the hypothalamus contains diverse, well-defined

Julia Maria dos Santos, Instituto de Neurociências \& Comportamento-INeC, Ribeirão Preto, SP, Brasil. Marcus Lira Brandão, Laboratório de Psicobiologia, Faculdade de Filosofia, Ciências e Letras de Ribeirão Preto, Universidade de São Paulo, Ribeirão Preto, SP, Brasil. Correpondence regarding this article should be directed to: Marcus Lira Brandão, Laboratório de Psicobiologia Faculdade de Filosofia, Ciências e Letras de Ribeirão Preto, Av. Bandeirantes, 3900, 14090-901 Ribeirão Preto-SP, Brazil, tel: 55-16-3602-3638, fax: 55-16-3602-4830, Email: mbrandao@usp.br nuclei that play a key role in the expression of the defense reaction (Canteras, 2002; Canteras et al., 1997; Canteras \& Blanchard, 2008).

These sites include a circuit formed by the anterior hypothalamic nucleus (AHN), dorsomedial portion of the ventromedial nucleus of the hypothalamus (VMHDM), dorsomedial hypothalamus (DMH), and dorsal premammillary nucleus (PMD), which has been termed the defensive hypothalamic area. This circuit is hypothesized to integrate innate defensive responses primarily involved in the processing of predatory threats (Canteras, 2002; Canteras et al. 1997; Johnson \& Shekhar, 2006). This neural substrate is also well known to be under tonic inhibitory control exerted by $\gamma$-aminobutyric acid (GABA) mechanisms (Brandão, et al., 1999; Brandão, Di Scala, Bouchet, \& Schmitt, 1986; Brandão, Zanoveli, Ruiz-Martinez, Oliveira, \& Landeira-Fernandez, 2008). A recent study using the fear-potentiated startle paradigm also showed that GABAergic mechanisms in the AHN and VMHDM regulate the neural systems that mediate conditioned fear (Santos, Macedo, \& Brandão, 2008). The AHN and PMD, but not VMHDM, appear to be involved in contextual conditioning to predator cues 
(Cezario, Ribeiro-Barbosa, Baldo, \& Canteras, 2008), but little research has been conducted to elucidate the involvement of these structures in classical conditioning paradigms using pairings of shock with a light or tone.

Given the importance of the hypothalamus in the generation of emotional states and in the expression of defensive behaviors, the present study explored the neural substrates underlying fear conditioning and assessed GABAergic mediation of conditioned fear in the medial hypothalamus, specifically the AHN and VMHDM, using fear conditioning to an explicit cue. In this paradigm, an emotionally neutral stimulus (e.g., light) is paired with an aversive unconditioned stimulus (US; e.g., footshock). The neutral stimulus thereby becomes a conditioned stimulus (CS) that elicits conditioned fear responses when subsequently presented alone during the expression phase of the experiment (Brandão et al., 2008).

To investigate the participation of GABAergic mechanisms in the hypothalamic nuclei associated with conditioned fear described above, the GABA receptor agonist muscimol and the GABA-synthesizing enzyme glutamic acid decarboxylase inhibitor semicarbazide were used as an enhancer and inhibitor of GABA mechanisms, respectively. Muscimol and semicarbazide were injected into the AHN and VMHDM of rats subjected to a conditioned fear paradigm and open-field test to detect drug effects related to enhancement of arousal or motor reactivity. The concentration of muscimol used in this study has been shown to have fear-reducing effects through interactions with $\mathrm{GABA}_{\mathrm{A}}$ receptors (Nobre \& Brandão, 2004). The concentration of semicarbazide used in this study has been shown to reduce local GABA transmission in limbic areas associated with the production of defensive behavior (Aguiar \& Brandão, 1994; Azami, Lewelyn, \& Roberts, 1980; Brandão et al., 1986).

\section{Materials and methods}

\section{Animals}

Fifty-six naive male Wistar rats from the animal house of the Campus of Ribeirão Preto, University of São Paulo, were used. The animals, weighing 250$280 \mathrm{~g}$ each, were housed in groups of four per cage under a $12 \mathrm{~h}: 12 \mathrm{~h}$ light/dark cycle (lights on at 07:00 h) at $22 \pm 1{ }^{\circ} \mathrm{C}$ with free access to food and water. The experiments were performed during the light phase of the light/dark cycle and were performed in compliance with the recommendations of SBNeC (Brazilian Society of Neuroscience and Behavior), which are based on the U.S. National Institutes of Health Guidelines for the Care and Use of Laboratory Animals.

\section{Surgery}

The animals were anesthetized with tribromoethanol $(250 \mathrm{mg} / \mathrm{kg}$, i.p.) and fixed in a stereotaxic frame with a flat skull position (David Kopf Instruments, Tujunga, CA, USA). One stainless-steel guide-cannula (length, $14 \mathrm{~mm}$; outer diameter, $0.6 \mathrm{~mm}$; inner diameter, $0.4 \mathrm{~mm}$ ) was implanted in the forebrain and aimed at the AHN or VMHDM. The upper incisor bar was set $-3.3 \mathrm{~mm}$ below the interaural line (skull horizontal between bregma and lambda). The guide-cannula was introduced vertically on the right side of the brain using the following coordinates, with bregma serving as the reference for each plane: AHN $(n=30$; anterior/posterior, 1.5 $\mathrm{mm}$; medial-lateral, $0.6 \mathrm{~mm}$; dorsal/ventral, $8.8 \mathrm{~mm}$ ), $\operatorname{VMHDM}(n=26$; anterior/posterior, $2.9 \mathrm{~mm}$; medial lateral, $0.5 \mathrm{~mm}$; dorsal/ventral, $9.3 \mathrm{~mm}$ ) (Paxinos \& Watson, 2005). The guide-cannula was fixed to the skull by means of acrylic resin and two stainless steel screws. At the end of the surgery, each guide-cannula was sealed with a stainless steel wire to protect it from blockage. Additionally, the animals received an intramuscular injection of Pentabiotic (60,000 IU, $0.2 \mathrm{ml}$; Fort Dodge Saúde Animal Ltda, Campinas, Brazil). The rats were then allowed a period of 1 week to recover from surgery.

\section{Apparatus and procedure}

\section{Training}

Animals were conditioned to a light CS in a cage $(20 \times 20 \times 25 \mathrm{~cm})$ with side and back walls constructed of stainless steel, and using transparent Plexiglas for the ceiling and front door. The grid floor of this cage consisted of stainless-steel rods spaced $1.5 \mathrm{~cm}$ apart. The cage was located within a ventilated and sound-attenuated chamber $(45 \times 45 \times 45 \mathrm{~cm})$. On each of 2 consecutive days, the animals were placed in the training cage, and 5 min later each rat received 10 CS-US pairings, with a $4 \mathrm{~s}$ light CS coterminating with a $1 \mathrm{~s}, 0.6 \mathrm{~mA}$ footshock US. The shocks were delivered through the floor of the training cage using a constant current generator equipped with a scrambler (Albarsh Instruments, Porto Alegre, Brazil). The CS was a white light emitted from a $6.0 \mathrm{~W}, 127 \mathrm{~V}$ bulb located in the ceiling of the chamber. Application of the stimulus was controlled by a microprocessor and an input/output board (Insight Equipment, Ribeirão Preto, Brazil). The intertrial interval varied randomly between $30 \mathrm{~s}$ and $120 \mathrm{~s}$. The duration of each training session was about $20 \mathrm{~min}$.

\section{Testing}

The testing sessions were conducted in a cage that was different from the one used for the training sessions. A larger cage $(48 \times 24 \times 30 \mathrm{~cm})$ with the lateral walls and ceiling made of white and opaque Plexiglas, respectively, was used only during testing sessions. The animals were placed in the testing cage, and after 5 min of habituation, animals were presented with the light CS only without any footshocks. Animal behavior was recorded with a video camera positioned behind the cage, with the signal relayed to a monitor in another 
room via a closed circuit. The criterion used to assess conditioned fear was the time that rats spent freezing during a 5-min period. Freezing was operationally defined as the total absence of movement of the animal, with the exception of respiration. The testing session was conducted similarly to the training sessions. The duration of the testing sessions was $10 \mathrm{~min}$.

\section{Open-field test}

Some of the animals that received microinjections of muscimol were subjected to the open-field test to assess locomotor activity. The test was performed in a circular arena made of Plexiglas (diameter, $60 \mathrm{~cm}$; height, 50 $\mathrm{cm}$ ), with the floor divided into 12 sections. The animals were first habituated to the open-field for $10 \mathrm{~min}$ prior to treatment. After treatment, animals were placed in the center of the arena and left for a 30-min period of free exploration. The number of crossings (i.e., number of floor sections traversed with four paws) was recorded every minute for 30 min by a video camera positioned above the arena.

\section{Drugs}

Muscimol hydrochloride (Sigma-Aldrich, São Paulo, Brazil) and semicarbazide hydrochloride (Vetec, São Paulo, Brazil) were dissolved in physiological saline $(0.9 \%)$ shortly before use. Physiological saline also served as vehicle control. Both muscimol (1 $\mathrm{nmol} / 0.2 \mu \mathrm{l})$ and semicarbazide $(6 \mu \mathrm{g} / 0.2 \mu \mathrm{l})$ were injected into the right side of the hypothalamus 10 and 20 min, respectively, before the testing session. Drug doses and times of injection were based on previous studies from this laboratory (Aguiar \& Brandão, 1994; Borelli, Ferreira-Netto, Coimbra, \& Brandão, 2005; Nobre \& Brandão, 2004; Santos et al., 2008). For the fear conditioning test, the number of animals was 30 for the AHN (saline, $\mathrm{n}=10$; muscimol, $\mathrm{n}=10$; semicarbazide, $\mathrm{n}=10$ ) and 26 for the VMHDM (saline, $\mathrm{n}=9$; muscimol, $\mathrm{n}=10$; semicarbazide, $\mathrm{n}=7$ ). For the open-field test, the number of animals was 18 for the AHN (saline, $\mathrm{n}=8$; muscimol, $\mathrm{n}=10$ ) and 13 for the VMHDM (saline, $\mathrm{n}=6$; muscimol, $\mathrm{n}=7$ ).

\section{Microinjection procedure}

To minimize the spread of the volume of injections, we used a glass micropipette for microinjections, according to a technique described elsewhere (Azami et al., 1980). Briefly, micropipettes were made of a fused silica capillary (outer diameter, $150 \mu \mathrm{m}$; inner diameter, $75 \mu \mathrm{m}$; Cluzeau Info Lab, Sainte Foy La Grande, France). To prevent broken cannulae, the capillary was fixed in a device comprised of $0.60 \mathrm{~mm} \times 25 \mathrm{~mm}$ and $1.00 \mathrm{~mm} \times 25 \mathrm{~mm}$ needles (BectonDickinson, Franklin Lakes, NJ, USA). In all surgeries, the guide-cannulae were positioned $6.0 \mathrm{~mm}$ below the skull. The fused silica capillary then protruded $2.8 \mathrm{~mm}$ and $3.3 \mathrm{~mm}$ beyond the guide-cannula to reach the $\mathrm{AHN}$ and
VMHDM, respectively. The micropipette was linked to a 5 $\mu 1$ Hamilton microsyringe by means of polyethylene tubing (PE-10; Becton-Dickinson) connected to a microinfusion apparatus (Harvard Apparatus, Holliston, MA, USA). A constant volume of $0.2 \mu \mathrm{l}$ was injected for $60 \mathrm{~s}$. The displacement of an air bubble inside the polyethylene tubing that connected the syringe needle to the glass needle was used to monitor the microinjections. Following the end of the injections, the microinjection pipettes were held inside the brain for 60 additional seconds to maximize diffusion away from the needle tip.

\section{Histology}

Upon completion of the experiments, $0.2 \mu \mathrm{l}$ of neutral red $(1 \%)$ was microinjected into the hypothalamic nuclei under study to mark the position of the microinjection sites. The animals were then given a lethal dose of chloral hydrate $(500 \mathrm{mg} / \mathrm{kg}$, i.p.) and perfused intracardially with $0.9 \%$ saline followed by $4 \%$ formalin solution. The brains were removed from the skulls and maintained in formalin solution for at least $2 \mathrm{~h}$. Brains were then cryoprotected in 30\% sucrose in $0.1 \mathrm{M}$ phosphate-buffered saline until soaked. Serial $60-\mu \mathrm{m}$ coronal brain sections were cut using a freezing microtome and mounted on gelatin-coated slides and stained with cresyl violet (5\%) (Sigma-Aldrich) to localize the positions of the microinjection sites according to the Paxinos and Watson atlas (2005). The microinjection sites were evaluated by microscopic examination. Rats with cannula misplacements or other cannula problems were excluded from the study.

\section{Statistical analysis}

Data are expressed as mean $\pm S E M$. For each anatomical location, the time of freezing during the entire session is expressed as mean \pm SEM. One-way analysis of variance (ANOVA) was used to assess the effects of muscimol and semicarbazide on groups of rats subjected to fear conditioning. Post hoc differences between group means were assessed with the NewmanKeuls test. Effects of treatments on the number of crossings in the open-field were assessed with Student's $t$-test. The significance level was set at $\mathrm{p}<0.05$.

\section{Results}

In our experimental conditions, pairings of footshocks with the light CS (4 s) caused a marked conditioned fear response comparable to that reported in other studies $(25,26)$. A photomicrograph of a representative injection site and the histological localization of representative injection sites in the AHN are shown in Figure 1A and B, respectively. The mean freezing time for animals that received microinjections of saline, muscimol, or semicarbazide into the $\mathrm{AHN}$ are shown in Figure 1C. One-way ANOVA revealed significant effects of treatment $(F[2,27]=8.85 ; p<0.05)$. 
Post hoc comparisons indicated that muscimol inhibited conditioned freezing in response to the light CS $(p<0.05)$. In contrast, semicarbazide enhanced this response $(p<0.05)$. As shown in Figure 1D, Student's $t$-test performed on the data obtained in the open-field test revealed no significant differences among treatments $(t[16]=0.71 ; p>0.05)$.
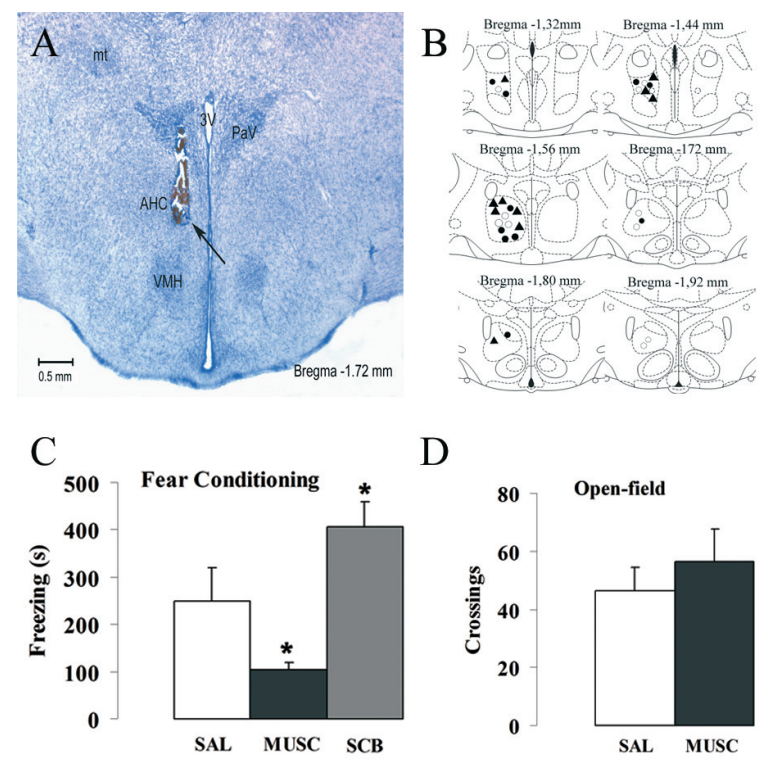

$\mathrm{D}$

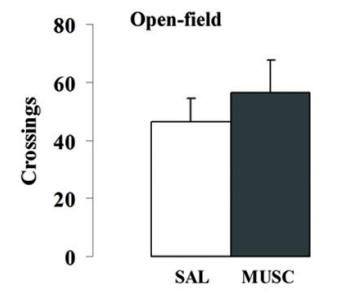

Figure 1. Photomicrograph of a representative site (A) and the location of microinjection sites $(\mathbf{B})$ in the anterior hypothalamic nucleus (AHN) with reference to the Paxinos and Watson atlas (2005). The arrow indicates the point of microinjection. Figures represent the atlas coordinates in $\mathrm{mm}$ posterior to bregma. ; saline $(0.9 \% \mathrm{NaCl}, \mathrm{SAL}, 0.2 \mu \mathrm{L}) ; \boldsymbol{\Delta}$, muscimol (MUSC, $1.0 \mathrm{nmol} / 0.2 \mu \mathrm{L}$ ); o, semicarbazide (SCB, $6.0 \mu \mathrm{g} / 0.2 \mu \mathrm{L}$ ). (C) Effects of intra-AHN administration of SAL, MUSC, or SCB on freezing time (s) exhibited by rats subjected to the fear conditioning procedure $(n=10$ for all groups) and on number of crossings (D) exhibited by rats subjected to the open-field test (saline, $n=8$; muscimol, $n=10$ ). Results are expressed as mean \pm SEM. ${ }^{*} p<.05$, compared with saline group (Newman-Keuls test). AHC, anterior hypothalamic area, central part; $\mathrm{PaV}$, paraventricular hypothalamic nucleus; VMH, ventromedial hypothalamic nucleus; $3 \mathrm{~V}$, third ventricle; $\mathrm{mt}$, mammillothalamic tract.

A photomicrograph of a representative injection site and the histological localization of representative injection sites in the VMHDM are shown in Figure $2 \mathrm{~A}$ and $\mathrm{B}$, respectively. Figure $2 \mathrm{C}$ displays the mean freezing time of animals that were subjected to lightCS fear conditioning and treated with saline, muscimol, or semicarbazide before the testing session. One-way ANOVA revealed significant effects of treatment $(F[2,23]=9.28 ; p<0.05)$. Post hoc comparisons indicated that muscimol inhibited conditioned freezing in response to the light CS $(p<0.05)$. As shown in Figure 2D, Student's $t$-test performed on the data in the open-field test revealed no significant differences between treatments $(t[16]=0.97 ; p>0.05)$.
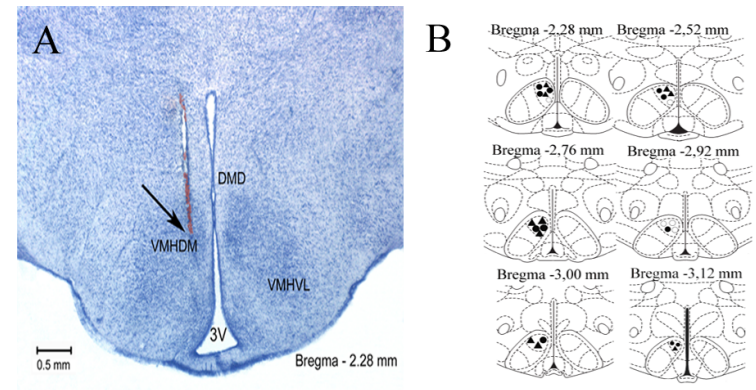

C

D
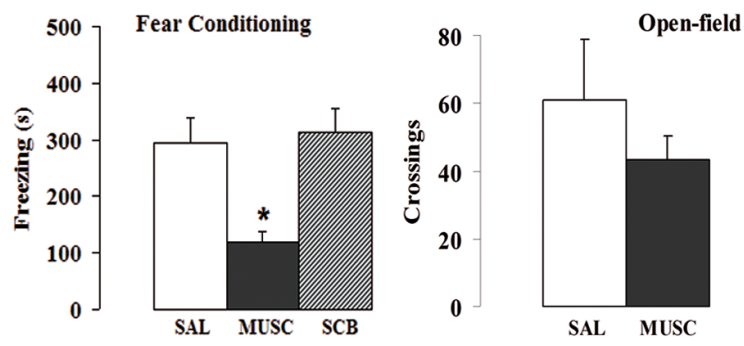

Figure 2. Photomicrograph of a representative site (A) and the location of microinjection sites $(\mathbf{B})$ in the dorsomedial part of the ventromedial hypothalamic nucleus (VMHDM) with reference to the Paxinos and Watson atlas (2005). The arrow indicates the point of microinjection. Figures represent the atlas coordinates in $\mathrm{mm}$ posterior to bregma. ', saline $(0.9 \%$ $\mathrm{NaCl}$, SAL, $0.2 \mu \mathrm{L}$ ); $\boldsymbol{\Delta}$, muscimol (MUSC, $1.0 \mathrm{nmol} / 0.2$ $\mu \mathrm{L}$ ); o, semicarbazide (SCB, $6.0 \mu \mathrm{g} / 0.2 \mu \mathrm{L})$. (C) Effects of intra-VMHDM administration of SAL $(n=9)$, MUSC $(n=$ $10)$, or SCB $(n=7)$ on freezing time (s) exhibited by rats subjected to the fear conditioning procedure and on the number of crossings (D) exhibited by rats subjected to the open-field test (saline, $n=6$; muscimol, $n=7$ ). Results are expressed as mean $\pm S E M .{ }^{*} p<.05$, compared with saline group (NewmanKeuls test). VMHDM, ventromedial hypothalamic nucleus, dorsomedial part; VMHVL, ventromedial hypothalamic nucleus, ventrolateral part; DMD, dorsomedial hypothalamic nucleus, dorsal part; $3 \mathrm{~V}$, third ventricle.

\section{Discussion}

The circuit formed by the AHN, DMH, VMHDM, and PMD comprises regions mainly activated by predatory encounters, supporting the existence of a segregated subsystem in the medial zone of the hypothalamus involved in the expression of innate fear responses (Canteras et al., 1997; Canteras, 2002). GABA antagonism in the medial hypothalamus generally induces defensive reactions, indicating tonic inhibitory role of this neurotransmitter in this brain region (Brandão et al., 1986; Milani \& Graeff, 1987; Schmitt, DiScala, Brandão, \& Karli, 1985).

Pharmacological impairment of GABAergic function in the DMH by chronic inhibition of local GABA synthesis revealed a pattern of defensive reactions that closely resembled the clinical sequelae of panic disorder (Johnson \& Shekhar, 2006). Moreover, chronic inhibition of GABA synthesis in the DMH has recently been shown to facilitate panic-like, lactateinduced changes in animals, an effect that was prevented 
by systemic administration of a selective group II metabotropic glutamate receptor agonist, which inhibits glutamate release (DiMicco, Samuels, Zaretskaia, \& Zaretsky, 2002; Jardim \& Guimarães, 2001). A recent study showed that intra-PMD administration of muscimol or semicarbazide did not change conditioned fear responses of rats subjected to fear-potentiated startle using a light CS (Santos et al., 2008). However, this finding does not necessarily refute the involvement of this hypothalamic nucleus in the conditioned fear response to other types of conditioned stimuli because the PMD was shown to exhibit the most striking increases in c-fos expression following exposure of rats to a cat (Canteras et al., 1997).

The present study showed that intra-AHN administration of the selective $\mathrm{GABA}_{\mathrm{A}}$ agonist muscimol significantly reduced the conditioned freezing response of rats subjected to the fear-conditioning paradigm using a light CS. Intra-AHN administration of the glutamic acid decarboxylase inhibitor semicarbazide, in contrast, significantly enhanced the conditioned response compared with the control group. The present results also showed that intra-VMHDM administration of muscimol reduced the conditioned fear responses of rats, but semicarbazide was without effect in this case. Similar results were obtained in a recent study from this laboratory, in which muscimol injected into the AHN and VMHDM also significantly reduced fear-potentiated startle in response to a light CS. In contrast, intra-VMHDM injection of semicarbazide did not significantly affect this response, but intra-AHN injection enhanced fear-potentiated startle. These differences in drug action may be related to their distinct modes of action. Whereas muscimol acts directly on GABAergic receptors located on postsynaptic neurons regardless of the main neurotransmitter present in their axon terminals, semicarbazide is a metabolic blocker that acts specifically on GABAergic neurons, which gradually reduces the pool of GABA to slowly promote defensive reactions (Aguiar \& Brandão, 1994; Santos et al., 2008). Thus, semicarbazide has specific actions on GABAergic neurons, whereas muscimol causes a more widespread inactivation of neurons in the injected region. A study aimed to establish the relative density of GABAergic neurons in the AHN/VMHDM could further clarify the distinct effects on conditioned freezing behavior obtained in the present study following injections of semicarbazide and muscimol into these hypothalamic nuclei.

The AHN and VMHDM comprise part of the medial hypothalamic zone, which plays a crucial role in the initiation of defensive behavior during a predatory encounter (Canteras, 2008; Canteras et al., 1997). Reciprocal anatomical projections are well known to exist between these nuclei (Risold, Canteras, \& Swanson, 1994). An intact AHN is required for the expression of defensive aggressive behavior elicited from the ventromedial nucleus of the hypothalamus
(Fuchs, Edinger, \& Siegel, 1985), and both nuclei receive inputs from telencephalic structures. The AHN integrates most of the septohippocampal inputs to the medial hypothalamic defensive system, and it could be an important recipient involved in mechanisms underlying learning and memory and sensory processing. The VMHDM can be regarded as a major recipient of fibers emerging from the corticomedial, basomedial, and basolateral nuclei of the amygdala (Risoldi et al., 1994). These hypothalamic nuclei, in turn, send information back to the cerebral cortex via the thalamus, and it is likely to initiate defensive responses via descending projections to brain stem regions, particularly the PAG (Canteras, 1997; McGregor, Hargreaves, Apfelbach, \& Hunt, 2004). The observed effects of muscimol in both structures (AHN and VMHDM) may be ascribed to a reduction of the conditioned freezing response of rats subjected to the fear-conditioning paradigm using a light CS and not to nonspecific effects because local injections of muscimol into these nuclei did not affect locomotor activity assessed by the open-field test.

Although much work still needs to be done to better understand the organization and chemistry of unconditioned and conditioned fear modulated by the medial zone of the hypothalamus, distinct subsets of neurons are likely to mediate different aspects of defensive responses. A previous study from this laboratory suggested that the $\mathrm{DMH}$ is more related to the organization of unconditioned fear responses to environmental threats, in which the dPAG plays a critical role (Brandão et al., 1986). Shock-based fear conditioning and defensive responses to predator exposure have also been reported to be mediated by distinct neural systems at the hypothalamus level (Canteras \& Blanchard, 2008). It has become clear that the systems mediating antipredatory defense and shock-based fear conditioning are processed by distinct neural circuits, not only at the hypothalamic level, but also in terms of the amygdalar processing. The information flow associated with shock-based fear conditioning reaches hypothalamic regions from the central nucleus of the amygdala (CEA). Efferent projections from the CEA to the brain stem and hypothalamic areas give rise to distinct behavioral and autonomic reactions involved in the expression of fear and anxiety (Davis, 1992). On the contrary, CEA lesions do not influence the defensive responses to either a predator (De Oca \& Fanselow, 2004) or its odor (Li, Maglinao, \& Takahashi, 2004). Moreover, the medial nucleus of the amygdala, and not the CEA, is important in processing predator cues (McGregor et al., 2004). The search for the output regions for the hypothalamic areas involved with defense reaction has produced some interesting findings. Evidence for the connectivity of these hypothalamic sites and midbrain tectum regions underlying anti-predatory defense has been provided systematically. For example, behavioral observations indicate that the PMD interferes 
with both unconditioned and conditioned antipredatory defensive behavior (Cezario, Ribeiro-Barbosa, Baldo, \& Canteras 2008). Moreover, cat odor-exposed rats showed Fos expression in the dorsal premammillary nucleus, periaqueductal grey, ventromedial hypothalamic nucleus (dorsomedial part), cuneiform nucleus and locus coeruleus (Cezario et al., 2008; Dielenberg, Hunt, \& McGregor, 2001; Staples, Hunt, Cornish, \& McGregor, 2005). However, still unclear is how the hypothalamic nuclei are interrelated in shock-based associative learning. Recent studies from this laboratory showed that the dPAG has neural elements associated not only with unconditioned fear but also with contextual conditioned fear (Brandão et al., 2008; Castilho \& Brandão, 2001; Castilho, Macedo, \& Brandão, 2002). The present study suggests that GABAergic mechanisms in the AHN and VMHDM are part of the regulatory mechanism of conditioned fear associated with a light CS.

\section{Conclusions}

Several studies have shown that the defensive hypothalamic zone is composed of the AHN, VMHDM, $\mathrm{DMH}$, and PMD, which are highly interconnected structures involved in integrating innate defensive responses to environmental threats (Canteras, 2002; Canteras \& Blanchard, 2008; Canteras et al., 1997). These regions contain groups of neurons that control unconditioned defensive behavior and are tonically inhibited by GABAergic neurotransmission (Brandão et al., 1986, 1999; Schmitt et al., 1986). The present study provides new data showing that the AHN and VMHDM are also recipients of GABA-mediated regulatory mechanisms involved in conditioned freezing elicited by pairings of a light CS with footshock.

\section{Acknowledgments}

Research was supported by FAPESP (Proc No. 11/00041-3). J.M. Santos holds a doctoral fellowship from FAPESP (Proc No. 11408-2/2008).

\section{References}

Aguiar, M.S. \& Brandão, M.L. (1994). Conditioned place aversion produced by microinjections of substance $\mathrm{P}$ into the periaqueductal gray of rats. Behavioural Pharmacology 5, 369-73.

Azami, J., Lewelyn, M.B., \& Roberts, M.H.T. (1980). An extra-fine assembly for intra-cerebral microinjection. Proceedings of the Physiological Society (UK), 18-19.

Brandão, M.L., Anseloni, V.Z., Pandóssio, J.E., De Araújo, J.E., \& Castilho, V.M. (1999). Neurochemical mechanisms of the defensive behavior in the dorsal midbrain. Neuroscience Biobehavioral Reviews 23, 863-75.

Brandão, M.L., Di Scala, G., Bouchet, M.J., \& Schmitt, P. (1986). Escape behavior produced by the blockade of glutamic acid decarboxilase $\mathrm{GAD}$ ) in mesencephalic central gray or medial hypothalamus. Pharmacology Biochemistry \& Behavior 24, 497-501.

Brandão, M.L., Zanoveli, J.M., Ruiz-Martinez, R.C., Oliveira, L.C., \& Landeira-Fernandez, J. (2008). Different patterns of freezing behavior organized in the periaqueductal gray of rats: association with different types of anxiety. Behavioural Brain Research 188, 1-13.

Borelli, K.G., Ferreira-Netto, C., Coimbra, N.C., \& Brandão, M.L. (2005). Fos-like immunoreactivity in the brain associated with freezing or escape induced by inhibition of either glutamic acid decarboxylase or GABA receptors in the dorsal periaqueductal gray. Brain Research 105, 100-11.

Canteras, N.S. (2002). The medial hypothalamic defensive system: hodological organization and functional implications. Pharmacology Biochemistry \& Behavior 71, 481-91.

Canteras, N.S., \& Blanchard, D.C. (2008). A behavioral and neural systems comparison of unconditioned and conditioned defensive behavior. In: R.J. Blanchard, D. Blanchard, G. Griebel, D. Nutt (Eds). Handbook of Anxiety and Fear (pp. 141-53). Amsterdam: Academic Press

Canteras, N.S., Chiavegatto, S, Ribeiro do Valle, L.E., \& Swanson, LW. (1997). Severe reduction of defensive behavior to a predator by discrete hypothalamic chemical lesions. Brain Research Bulletin 44, 297-305.

Castilho, V.M., \& Brandão, M.L. (2001). Conditioned antinociception and freezing using electrical stimulation of the dorsal periaqueductal gray or inferior colliculus as unconditioned stimulus are differentially regulated by $5-\mathrm{HT} 2 \mathrm{~A}$ receptors in rats. Psychopharmacology 155, 154-62.

Castilho,V.M., Macedo, C.E., \& Brandão, M.L. (2002). Role of benzodiazepine and serotonergic mechanisms in conditioned freezing and antinociception using electrical stimulation of the dorsal periaqueductal gray as unconditioned stimulus in rats. Psychopharmacology, 165, 77-85.

Cezario, A.F., Ribeiro-Barbosa, E.R., Baldo, M.V., \& Canteras, N.S. (2008). Hypothalamic sites responding to predator threats: the role of the dorsal premammillary nucleus in unconditioned and conditioned antipredatory defensive behavior. European Journal of Neuroscience 28, 1003-15.

Davis, M. (1992). The role of the amygdala in fear and anxiety. Annual Review of Neuroscience 15, 353-75.

De Oca, B.M., \& Fanselow, M.S. (2004). Amygdala and periaqueductal gray lesions partially attenuate unconditional defensive responses in rats exposed to a cat. Integrative Physiology Behavioral Science 39, 318-33.

Dielenberg, R.A., Hunt, G.E., \& McGregor, I.S. (2001). "When a rat smells a cat": the distribution of Fos immunoreactivity in rat brain following exposure to a predatory odor. Neuroscience 104, 1085-97.

DiMicco, J.A., Samuels, B.C., Zaretskaia, M.V., \& Zaretsky, D.V. (2002). The dorsomedial hypothalamus and the response to stress: part renaissance, part revolution. Pharmacology Biochemistry \& Behavior 71, 469-80.

Fuchs, S.A.G., Edinger, H.M., \& Siegel, A. (1985). The role of the anterior hypothalamus in affective defense behavior elicited from the ventromedial hypothalamus of the cat. Brain Research 330, 93-107.

Graeff, F.G. (2004). Serotonin, the periaqueductal gray and panic. Neuroscience Biobehavioral and Reviews 28, 239-59.

Jardim, M.C., \& Guimarães, F.S. (2001). GABAergic and glutamatergic modulation of exploratory behavior in the dorsomedial hypothalamus. Pharmacology of Biochemistry \& Behavior 69, 579-84.

Johnson, .P.L., \& Shekhar, A. (2006). Panic-prone state induced in rats with GABA dysfunction in the dorsomedial hypothalamus is mediated by NMDA receptors. Journal of Neuroscience 26, 7093-104.

Li, C.I., Maglinao, T.L., \& Takahashi, L.K. (2004). Medial amygdala modulation of predator odor induced unconditioned fear in the rat. Behavioral Neuroscience 118, 324-32.

McGregor, I.S., Hargreaves, G.A., Apfelbach, R., \& Hunt, G.E. (2004). Neural correlates of cat odor-induced anxiety in rats: region-specific effects of the benzodiazepine midazolam. Journal of Neuroscience 24, 4134-44.

Milani, H., \& Graeff, F.G. (1987). GABA-benzodiazepine modulation of aversion in the medial hypothalamus of the rat. Pharmacology of Biochemistry \& Behavior 28, 21-7.

Nobre, M.J., \& Brandão, M.L. (2004). Analysis of freezing behavior and ultrasonic vocalization in response to foot-shocks, ultrasound signals and GABAergic inhibition in the inferior colliculus: effects of muscimol and midazolam. European Neuropsychopharmacology 14, 45-52.

Paxinos, G., \& Watson, C. (2005). The Rat Brain in Stereotaxic Coordinates (5th edition), San Diego: Academic Press.

Risold, P.Y., Canteras, N.S., \& Swanson, L.W. (1994). Organization of projections from the anterior hypothalamic nucleus: a Phaseolus 
vulgaris-leucoagglutinin study in the rat. Journal of Comparative Neurology 348, 1-40.

Santos, J.M., Macedo, C.E., \& Brandão, M.L. (2008). Gabaergic mechanisms of hypothalamic nuclei in the expression of conditioned fear. Neurobiology of Learning \& Memory 90, 560-568.

Schmitt, P., DiScala, G., Brandão, M.L., \& Karli, P. (1985). Behavioral effects of microinjections of SR95103, a new GABA antagonist, into medial hypothalamus or the mesencephalic central gray. European Journal of Pharmacology 117, 149-58.

Staples, L.G., Hunt, G.E., Cornish, J.L., \& McGregor, I.S. (2005).

Neural activation during cat odor-induced conditioned fear and 'trial 2' fear in rats. Neuroscience Biobehavioral Reviews 29, 1265-77. 